Lateral distribution of pulse height in a parallel-plate avalanche counter

C. Y. Wu, D. Cline, E. Kwan, A. Chyzh, A. Hayes, I. Y. Lee, D. Swan

May 17, 2011 
This document was prepared as an account of work sponsored by an agency of the United States government. Neither the United States government nor Lawrence Livermore National Security, LLC, nor any of their employees makes any warranty, expressed or implied, or assumes any legal liability or responsibility for the accuracy, completeness, or usefulness of any information, apparatus, product, or process disclosed, or represents that its use would not infringe privately owned rights. Reference herein to any specific commercial product, process, or service by trade name, trademark, manufacturer, or otherwise does not necessarily constitute or imply its endorsement, recommendation, or favoring by the United States government or Lawrence Livermore National Security, LLC. The views and opinions of authors expressed herein do not necessarily state or reflect those of the United States government or Lawrence Livermore National Security, LLC, and shall not be used for advertising or product endorsement purposes.

This work performed under the auspices of the U.S. Department of Energy by Lawrence Livermore National Laboratory under Contract DE-AC52-07NA27344. 
LLNL-TR-pending

\title{
Lateral distribution of pulse height in a parallel-plate avalanche counter
}

\author{
C.Y. Wu ${ }^{1}$, D. Cline ${ }^{2}$, E. Kwan ${ }^{1}$, A. Chyzh ${ }^{1}$, A. Hayes ${ }^{2}$, I.Y. Lee ${ }^{3}$, and D. Swan ${ }^{4}$ \\ 1. Lawrence Livermore National Laboratory, Livermore, CA 94550 \\ 2. Department of Physics, University of Rochester, Rochester, NY 14627 \\ 3. Lawrence Berkeley National Laboratory, Berkeley, CA 94720 \\ 4. Swan Research LLC, Owosso, MI 48867
}

\section{Introduction}

The nuclear $\gamma$-ray spectroscopy enters a new era when the first engineering run of GRETINA [1], a new generation of $\gamma$-ray tracking arrays, was successfully carried out at LBNL in early April, 2011. A parallel effort has been setup to develop the auxiliary charged-particle detector arrays with a matching position resolution to fully exploit the potential of GRETINA. Improving the position resolution of the existing charged-particle detector array, $\mathrm{CHICO}$ [2], is a part of this coordinated effort.

CHICO has been proven to be a valuable auxiliary charged-particle detector array for Gammasphere [3]. It is a highly segmented parallel-plate avalanche counter with position resolution of $\sim 1^{\circ}$ in $\theta$ and $\sim 9^{\circ}$ in $\phi$ and solid-angle coverage of $69 \%$ of $4 \pi$. Over the period between 1996 and 2008, a total of 26 experiments were fielded successfully, involving 58 experimentalists from 17 institutions, which results in 37 publications and 5 Ph.D.'s.

The proposal to upgrade CHICO was approved by DOE/SC at the end of FY10. The goal is to redesign the cathode board and amplifier to improve the resolution for both $\theta$ and $\phi$ coordinates to better than one degree to match that of GRETINA. This project is scheduled to complete by the summer of 2012 .

\section{Technical approaches}

We proposed to improve the CHICO position resolution by adding a $\phi$ sensing in addition to the existing $\theta$ sensing for the cathode by pixelating the board. Pixels are interconnected by next to the adjacent ones in two orthogonal coordinates, shown in Fig. 1, and then connected to the delayed line. Therefore, the actual position is not determined by the pixel itself rather by the time readout from the delay line.

The success of this delay-line readout technique has been demonstrated by using a test board with $31 \times 31$ pixels connected 310 -ns delay chips $(1 \mathrm{~ns} / \mathrm{tap})$ for each coordinate, which was designed and fabricated by Swan Research. Each pixel has a dimension of $1.6 \times 1.6 \mathrm{~mm}$ with $0.4 \mathrm{~mm}$ gap between them. Excellent uniformity and linearity were achieved and shown in Fig. 2 and 3, respectively.

Since the position sensing for CHICO is made in the polar coordinate, the pixel size is varied depending on the angle. Therefore, to optimize the pixelated board design for CHICO, it is 
important to know the lateral distribution of pulse height in a parallel-plate avalanche counter. For this purpose, a test board with a string of 21 electrically insulated pixels was designed and fabricated. Each pixel has a dimension of $0.9 \times 0.9 \mathrm{~mm}$ with a gap of $0.2 \mathrm{~mm}$ between them. The measurement was carried out using a collimated ${ }^{252} \mathrm{Cf}$ source on a single pixel and the pulse heights of this pixel together with adjacent pixels were recorded simultaneously by a digital scope. The experimental setup is shown in Fig. 4. The results together with the calculation are given in Fig. 5, where the agreement is surprising good. The calculation was done by one of us (D.C.) and is given in the appendix. Examples of the signal trace are shown in Fig.6, indicating the rise time $~ 3.5-4$ ns.

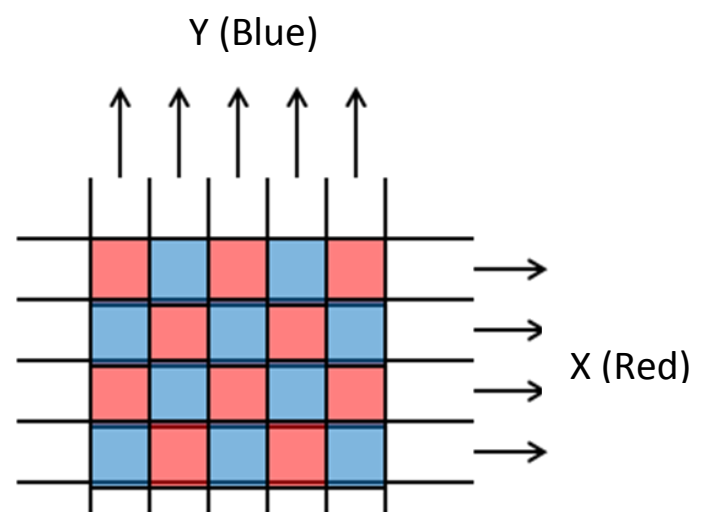

Fig. 1 Schematic view of the pixel interconnection electrically. $X$ position is readout from the electrically connected red pixels in horizontal direction. Y position is from the blue pixels in vertical direction.
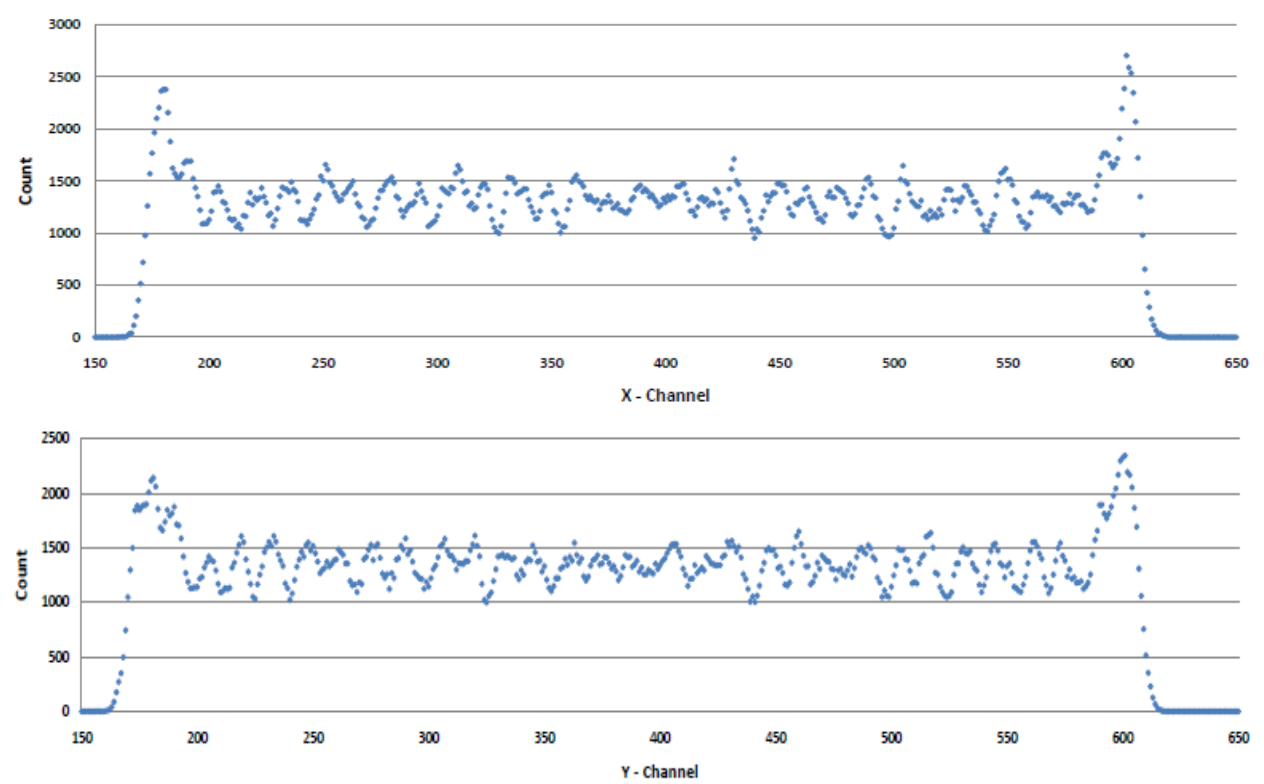

Fig. 2 The position measurement by taking the time difference between two ends of the delay line. Remarkable uniformity in both coordinates is achieved. 

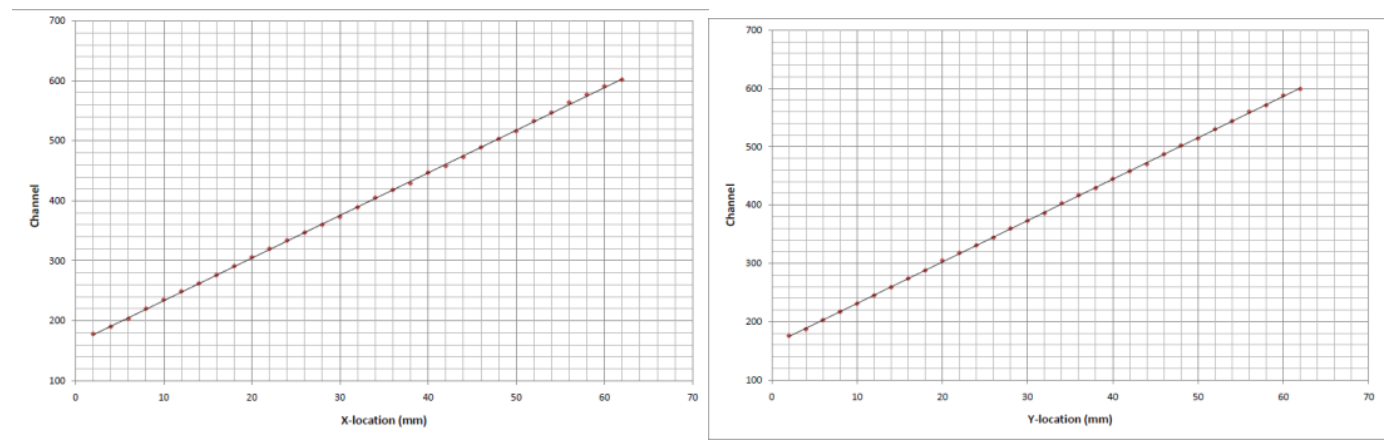

Fig. 3 The channel number vs. the relative position in $\mathrm{mm}$. Excellent linearity for both coordinates is achieved.

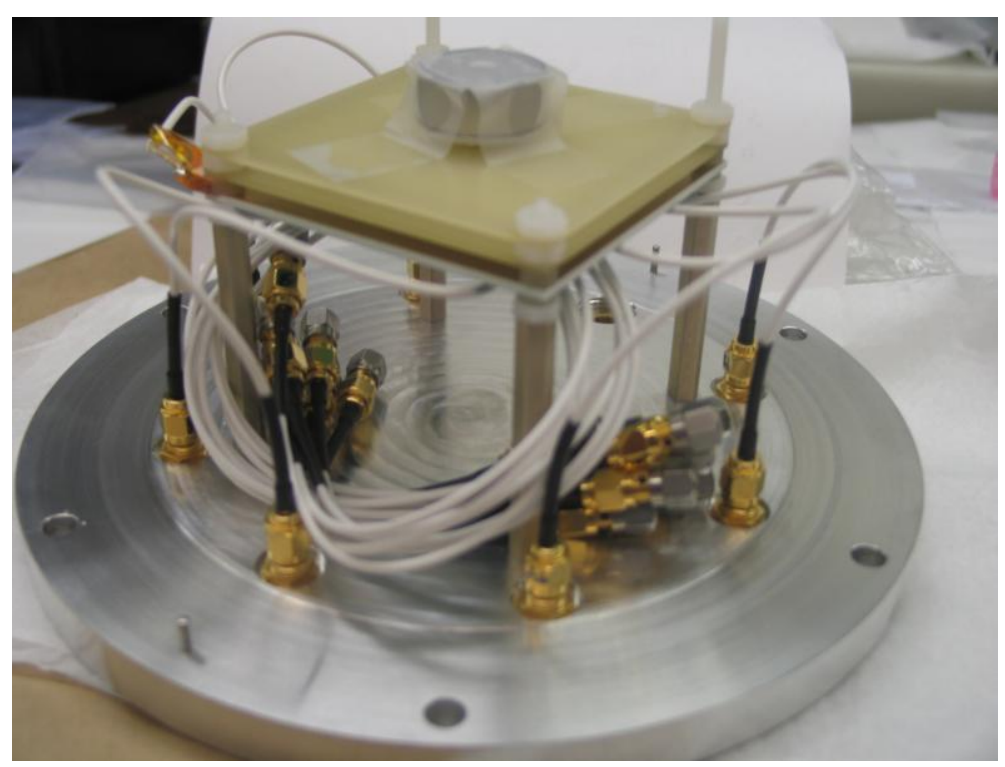

Fig. 4 The experimental setup for the measurement of pulse height distribution in a parallel-plate avalanche counter. A collimated ${ }^{252} \mathrm{Cf}$ source can be seen on the top of this setup.

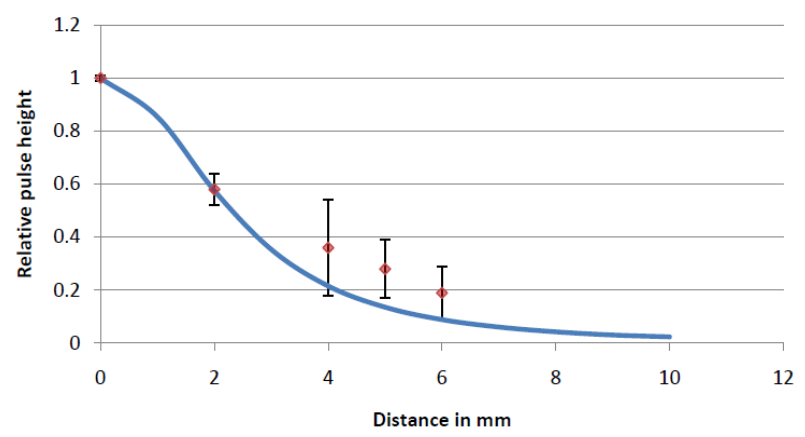

Fig. 5 The comparison of the lateral distribution of pulse height in a parallelplate avalanche counter between the measurement and calculations. 


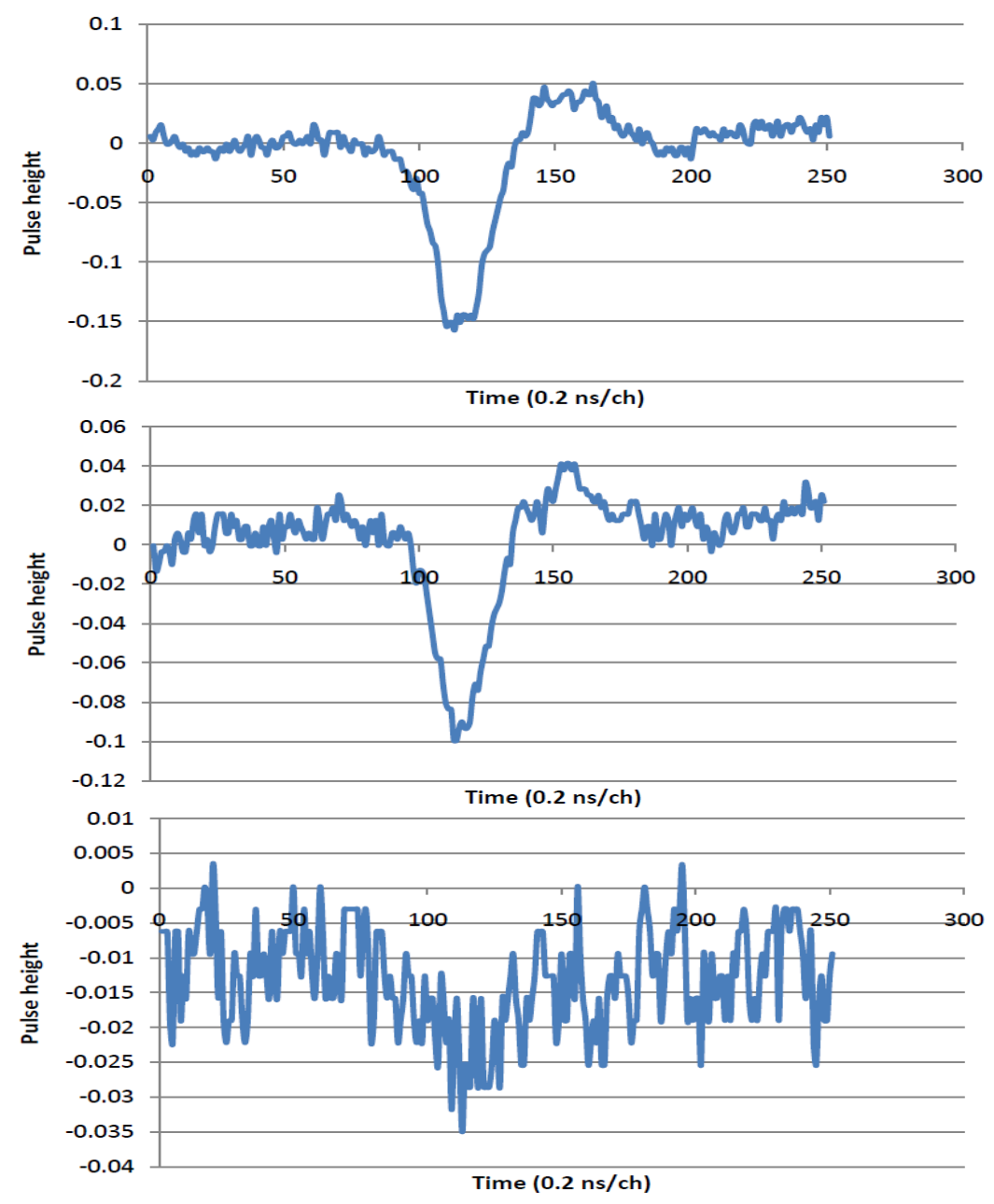

Fig. 6 The pulse shapes from the parallel-plate avalanche counter at a distance 0,2 , and $4 \mathrm{~mm}$ (top to bottom) away from the event location.

\section{Summary}

We have learned valuable lessons from these tests on the design, fabrication, and operation of the pixelated board with the delay-line readout technique for the parallel-plate avalanche counter. In addition to the fully pixelated board in both $\theta$ and $\phi$ coordinates, one of us (D.C.) proposed a hybrid board using the full strip for the $\theta$-sensing and the pixel for the $\phi$-sensing. To learn if a significant disparity in the pulse height between $\theta$ and $\phi$ from an avalanche, a test board with a similar pattern as the proposed hybrid board, shown in Fig. 7, will be designed and fabricated. This work is in progress.

After this test, a decision will be made on the final board design. The schedule to complete the design and fabrication of this pixelated board in addition to the amplifier will be the end of CY2011. 


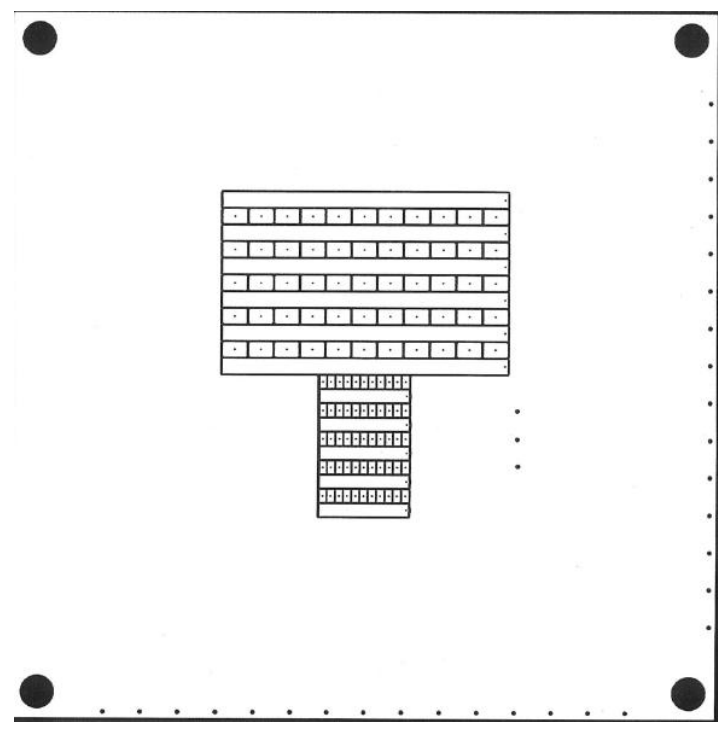

Fig. 7 The test board for the sandwich geometry design, where the $\mathrm{X}$-coordinate is readout from the horizontal band and the $Y$ coordinate is readout from the pixels connected vertically.

\section{References:}

[1] http://grfs1.lbl.gov

[2] M.W. Simon, D. Cline, C.Y. Wu, R.W. Gray, R. Teng, and C. long, "CHICO, a heavy ion detector for Gammasphere", Nucl. Instrum. Methods in Phys. Res. A452, 205 (2000) [3] I.Y. Lee, Proceedings of the Workshop on Gammasphere Physics, Berkeley, CA, Edited by M.A. Deleplanque, I.Y. Lee, and A.O. Macchiavelli (World Scientific, Singapore, 1996), p.50. 
TO: $\quad$ Ching-Yen Wu, I-Yang Lee, Adam Hayes, Dennis Swan, Bob Flight

FROM: Doug Cline

\section{RE: $\quad$ Thoughts on the design of $\mathrm{CHICO} 2$}

DATE: 23 February 2010

Our discussions of CHICO2 at the FRIB Workshop were very fruitfull and stimulated me to think more about the various design issues. Since I spent 10 hours at Detroit Metro airport trying to get a plane home on Monday gave me plenty of time to think about CHICO2. The following are some of my thoughts.

\subsection{Spatial distribution of the induced cathode signal.}

The fast PPAC signal comes from the electron shower that is maximal at the anode surface with maximum contribution from ionization near the cathode. The positive charges move much more slowly $(\approx \mu s)$ and their influence is heavily suppressed by the short differential time constant. Thus we can model having a negative charge near the anode. If we ignore image charges from the anode foil, then the electron charge, plus the positive image charge in the cathode, produce an electric field at the cathode that is given by the classic field of an electric dipole with the plane equipotential surface being perpendicular and equidistant from the charge $Q$ a distance $z=+D$ above the $(x, y)$ cathode plane and the positive image charge a distance $-D$ behind the cathode plane. That is, the electric field $E$ at the cathode a location $r$ from the perpendicular from the cathode to the induced charge is

$$
\mathbf{E}=\frac{Q D}{2 \pi \epsilon_{0}\left(D^{2}+r^{2}\right)^{\frac{3}{2}}}
$$

The $E$ field points out of the cathode plane and leads to a positive induced surface charge density on the cathode of

$$
\begin{aligned}
\sigma & =\epsilon_{0} E \\
& =\frac{Q D}{2 \pi\left(D^{2}+r^{2}\right)^{\frac{3}{2}}}
\end{aligned}
$$

This is evaluated in the table 1 for $D=3 m m$, where $r$ in $m m$ is the radial distance from the electric dipole axis in the surface of the cathode.

Table 1: Calculated surface charge density induced on the cathode for $3 \mathrm{~mm}$ spacing.

\begin{tabular}{|c|c|c|c|}
\hline$r(\mathrm{~mm})$ & Surface charge density $\sigma\left(\mathrm{C} / \mathrm{mm}^{2}\right)$ & Relative surface charge density & Fraction of enclosed charge for $\leq r$ \\
\hline 0 & 0.1111 & 1. & 0 \\
\hline 1 & 0.0948 & 0.854 & 0.0513 \\
\hline 2 & 0.0640 & 0.576 & 0.1680 \\
\hline 3 & 0.0392 & 0.354 & 0.2929 \\
\hline 4 & 0.0240 & 0.216 & 0.4000 \\
\hline 5 & 0.0151 & 0.136 & 0.4855 \\
\hline 6 & 0.0099 & 0.089 & 0.5528 \\
\hline 7 & 0.0068 & 0.061 & 0.6061 \\
\hline 8 & 0.0048 & 0.043 & 0.6489 \\
\hline 9 & 0.0035 & 0.031 & 0.6838 \\
\hline 10 & 0.0026 & 0.024 & 0.7127 \\
\hline
\end{tabular}


If this naive model is satisfactory then $55 \%$ of the charge is deposited within a $6 \mathrm{~mm}$ radius, which corresponds to the spacing between the pair of electric dipole charges. As given below, this charge distribution can be integrated over the individual pixels to to get the relative induced charge on each pixel. If the above calculations are valid then a suggested pixel dimension of $\leq 2 \mathrm{~mm}$ appears desirable for reliable cathode sensing as well as to match the performance of GRETINA.

\subsection{Suggested pixel size and geometry}

The size of the pixels, assuming $1^{\circ}$ spacing for both $\theta$ and $\phi$, is given in $m m$ in table 2 .

Table 2: CHICO2 pixel dimensions assuming $1^{\circ}$ spacing at $d=128 \mathrm{~mm}$ with normal at $\theta=49^{\circ}$.

\begin{tabular}{|c|c|c|}
\hline$\theta$ & $\theta$ slice width $m m$ & $\phi$ slide width $m m$ \\
\hline 12 & 3.51 & 0.58 \\
\hline 15 & 3.26 & 0.70 \\
\hline 20 & 2.93 & 0.88 \\
\hline 25 & 2.68 & 1.04 \\
\hline 30 & 2.50 & 1.18 \\
\hline 35 & 2.37 & 1.32 \\
\hline 40 & 2.29 & 1.46 \\
\hline 45 & 2.24 & 1.59 \\
\hline 50 & 2.23 & 1.71 \\
\hline 55 & 2.26 & 1.84 \\
\hline 60 & 2.32 & 1.97 \\
\hline 65 & 2.42 & 2.11 \\
\hline 70 & 2.57 & 2.25 \\
\hline 75 & 2.77 & 2.40 \\
\hline 80 & 3.05 & 2.57 \\
\hline 85 & 3.42 & 2.76 \\
\hline
\end{tabular}

The above pixel widths of up to $3.5 \mathrm{~mm}$ for $\theta$ are as large as the PPAC gap and thus could be important both for GRETINA Doppler correction as well as cathode sensing. The $\phi$ width is not a problem since it is only $>2 \mathrm{~mm}$ at $\theta>65^{\circ}$ where the recoil velocities, and concomitant Doppler broadening, are small.

I have simulated the response for two geometries assuming that only the electron charge at $D=3 \mathrm{~mm}$ plus the mirror charge in the cathode plane at $-D$ contribute to the charge distribution. That is, the image charges in the anode foil are neglected since the electric dipole moment for this anode image charge should be negligible due to the negative charge distribution being so close to the anode..

A) Checker-Board geometry, $\Delta \theta=\Delta \phi=1^{\circ}$

For the checker-board style geometry discussed so far, the simulations predict that at $\theta=60^{\circ}$ the pulse height will fluctuate between $50.17 \%$ and $49.15 \%$ depending on the location of the ionization relative to the pixels, that is, there is a $2.1 \%$ fluctuation in pulse height. This is acceptable.

B) Sandwich board, $\Delta \theta=\Delta \phi=1^{\circ}$

Initially I thought that the fluctuations would be larger than calculated above. One solution to this would be to subdivide the pixels into half degree widths but this will result in a rediculously large number of delay line taps and vias that are not required by the physics. Therefore, I propose a new geometry for the pixels on the cathode board where the $\theta$ slices have dimensions $\Delta \theta=0.5^{\circ}$ by $\Delta \phi=28^{\circ}$ with spacing of $1^{\circ}$ between each $\theta$ slice. That is, each narrow $\theta$ slice is the full $\phi$ width of the board and they are separated by the segmented $\phi$ slices that each have $\Delta \theta=0.5^{\circ}$ and $\Delta \phi=1^{\circ}$. This halves the minimum spacing between the $\theta$ and $\phi$ slices to minimize dependence on location of the ionization. For the sandwich board geometry the same simulation as above predicts a variation in the ratio of the maximum to minimum pulse height of $0.8 \%$ which is about a third of the case for the checker board, but neither of them is that large according to my simulations. The sandwich board geometry requires essentially the same number of vias and taps as the 


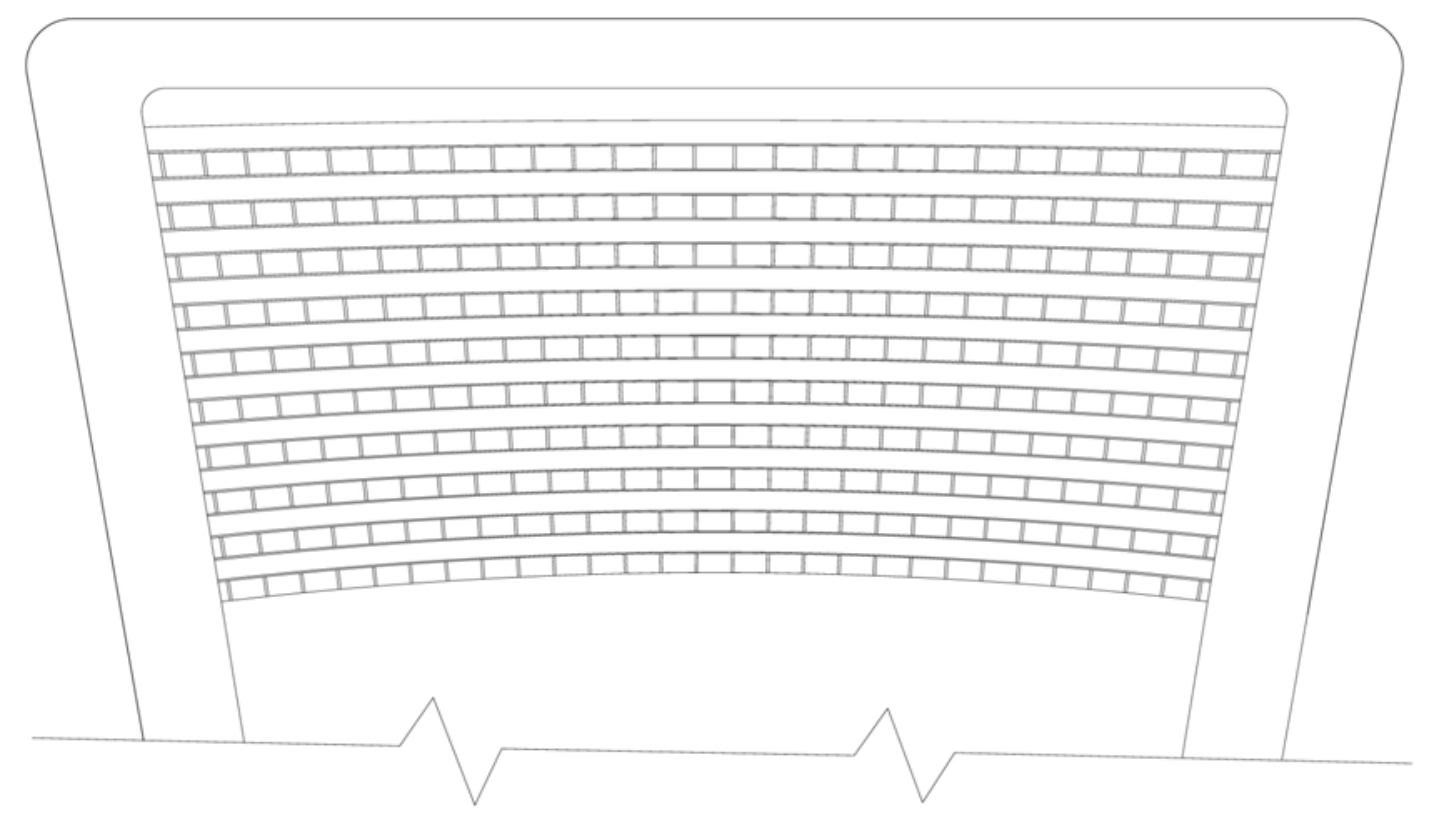

Figure 1: Sandwich geometry cathode layout

checker board option. The sandwich board geometry is capable of giving a slightly better $\theta$ resolution and better defines the angle $\theta$, but I suspect the difference is small. I like the sandwich board geometry because it will be easy to implement reducing the number of phi slices at small $\theta$.

\subsection{Required tasks;}

\section{1) Measure the cathode charge distribution:}

Ching-Yen; it is especially important that the charge distribution on the cathode be measured since CHICO2 is designed upon the assumption that we can simultaneously measure $\theta$ and $\phi$ with $100 \%$ efficiency. The $2 \mathrm{~mm}$ spacing between the pixels of the rectangular prototype board is ideally suited to measure the charge distribution for the cathode. I suggest that the outputs of 4 pixels along one line be connected to individual output cables and the ratio of each pixel pulse height to anode pulse height be recorded for different location of the collimated source to map the charge distribution and compare with the above simulation predictions.

2) Predicted performance of CHICO2:

I-Yang; it would be nice to know the predicted energy resolution for GRETINA and segmented Gammasphere assuming that $\mathrm{CHICO} 2$ has angular resolution of $\Delta \theta=\Delta \phi=1^{\circ}$.

3) Design cathode board:

Please let me know your thoughts regarding checker board versus sandwich board geometry and then Bob and I can start the detailed drafting of the cathode board geometry to send to Dennis. A sketch of the proposed sandwich board geometry is given below. 